\title{
BARYON CHIRAL PERTURBATION THEORY A.D. 1994
}

\author{
Ulf-G. Meißner ${ }^{\dagger, \star}$ \\ †Universität Bonn, Institut für Theoretische Kernphysik, Nussallee 14-16, \\ D-53115 Bonn, Germany \\ *email: meissner@pythia.itkp.uni-bonn.de
}

\begin{abstract}
In these lectures, the status of baryon chiral perturbation theory is reviewed. Particular emphasis is put on the two-flavor sector and the physics related to electromagnetic probes. I discuss in some detail the structure of the effective Lagrangian at next-to-leading order, the meaning of low-energy theorems in Compton scattering and pion photoproduction and confront the chiral predictions with the existing data. Some remaining problems and challenges are outlined.
\end{abstract}

Lectures given at the Indian Summer School on Electron Scattering off Nucleons and Nuclei, Prague, Czech Republic, September 1994

TK 9417

November 1994 


\section{Introduction}

Over the last few years, a tremendous amount of very precise data probing the structure of the nucleon at low energies has become available. Just to name a few, these are the measurements of neutral pion photo- and electroproduction in the threshold region [1] [2] [3] or the determinations of the nucleon electromagnetic polarizabilities at Illinois, Saskatoon, Oak Ridge and Mainz [4] [5] [6] [7]. While the interpretation of some of these data has been controversial, it is unchallenged that they encode information about the nucleon in the non-perturbative regime, i.e. at typical momentum scales were straightforward perturbation theory in the running strong coupling constant $\alpha_{S}\left(Q^{2}\right)$ is no longer applicable. At present, there exist essentially two approaches to unravel the physics behind the wealth of empirical information. On one hand, one uses models which stress certain aspects of the strong interactions (but tend to neglect or forget about others) like e.g. the constituent quark model presented here by Buchmann [8]. The other possibilitiy is to make use of the symmetries of the Standard Model (SM) and formulate an effective field theory (EFT) to systematically explore the strictures of these symmetries. In the case of QCD and in the sector of the three light quarks $\mathrm{u}, \mathrm{d}$ and $\mathrm{s}$ we know that there exist an approximate chiral symmetry which is spontaneously broken with the apperance of nine Goldstone bosons, the pions, kaons and the eta. These pseudoscalar mesons are the lightest strongly interacting particles and their small but non-vanishing masses can be traced back to the fact that the current masses $m_{u}, m_{d}$ and $m_{s}$ are small compared to any typical hadronic scale, like e.g. the proton mass. In the meson sector, the EFT is called chiral perturbation theory and is well developed and applied successfully to many reactions [9] [10] (for a review, see [11]). Notice that Ecker has given a nice introduction to this topic at this school last year [12] so I will try to avoid too much overlap with his lectures. Of course, there is also the lattice formulation of QCD which over the years has shown great progress but is not yet in the status to discuss in detail what will be mostly the topic here, namely the baryon structure as accurately probed with real or virtual photons. Clearly, the baryons (and in particular the nucleons I will mostly focus on) are not related directly to the spontaneous chiral symmetry breakdown. However, their interactions with pions and among themselves are strongly constrained by chiral symmetry. This is, of course, known since the sixties (see e.g. the lectures by Coleman [13] and references therein). However, to go beyond the current algebra or tree level calculations, one needs a systematic power counting scheme as it was first worked out for the meson sector by Weinberg [14]. As shown by Gasser et al. [15], the straightforward generalization to the baryon sector leads to problems related to the non-vanishing mass of the baryons in the chiral limit, i.e. one has an extra large mass scale in the problem. Stated differently, baryon four-momenta are never small compared to the chiral symmetry breaking scale $\Lambda_{\chi}, m_{B} / \Lambda_{\chi} \sim 1$. This can be overcome by a clever choice of velocity-dependent fields [16] 
which allows to transform the baryon mass term in a string of $1 / m_{B}$ suppressed interaction vertices. Then, a consistent power counting scheme emerges where the expansion in small momenta and quark masses can be mapped one-to-one on a (Goldstone boson) loop expansion.

These lectures will be organized as follows. After a short review of the construction of the EFT in the presence of matter fields, I will give a sample calculation (of the pion cloud contribution to the nucleon mass). I elaborate in some detail on the structure of the next-to-leading order terms of dimension two in the effective Lagrangian. In particular, I show how certain operators with fixed coefficients arise when the baryons are treated in the extreme non-relativistic limit. This is followed by a study of the numerical values of the low-energy constants appearing at this order. Then, I will discuss the status of the low-energy theorems in pion photo- and electroproduction and Compton scattering. In section 7 , chiral predictions are confronted with the data and the problems and open questions are adressed in section 8.

\section{Chiral perturbation theory with baryons}

Chiral perturbation theory (CHPT) is the EFT of the SM at low energies in the hadronic sector. Since as an EFT it contains all terms allowed by the symmetries of the underlying theory [14, it should be viewed as a direct consequence of the SM itself. The two main assumptions underlying CHPT are that

(i) the masses of the light quarks $\mathrm{u}, \mathrm{d}$ (and possibly $\mathrm{s}$ ) can be treated as perturbations (i.e., they are small compared to a typical hadronic scale of $1 \mathrm{GeV}$ ) and that

(ii) in the limit of zero quark masses, the chiral symmetry is spontaneously broken to its vectorial subgroup. The resulting Goldstone bosons are the pseudoscalar mesons (pions, kaons and eta).

CHPT is a systematic low-energy expansion around the chiral limit [14] [9] [10] [17]. It is a well-defined quantum field theory although it has to be renormalized order by order. Beyond leading order, one has to include loop diagrams to restore unitarity perturbatively. Furthermore, Green functions calculated in CHPT at a given order contain certain parameters that are not constrained by the symmetries, the so-called low-energy constants (LECs). At each order in the chiral expansion, those LECs have to be determined from phenomenology (or can be estimated with some model dependent assumptions). For a review of the wide field of applications of CHPT, see, e.g., Ref. [11.

In the baryon sector, a complication arises from the fact that the baryon mass $m_{B}$ does not vanish in the chiral limit [15]. Stated differently, only baryon three-momenta can be 
small compared to the hadronic scale. To see this in more detail, let us consider the two-flavor case with $m_{u}=m_{d}=\hat{m}$ and collect the proton and the neutron in a bi-spinor

$$
\Psi=\left(\begin{array}{l}
p \\
n
\end{array}\right)
$$

which transforms non-linearly under chiral transformations,

$$
\Psi \rightarrow \Psi^{\prime}=K(L, R, U(x)) \Psi
$$

Here, $K(L, R, U(x))$ is a non-linear function of the meson fields collected in $U(x)$ and of $L, R \in \mathrm{SU}(2)$ [18] [19]. It is defined via

$$
u^{\prime}=L u K^{\dagger}=K u R^{\dagger}, \text { with } U=u^{2}, u^{\prime 2}=U^{\prime 2} .
$$

The unimodular unitary matrix $U\left(U^{\dagger} U=U U^{\dagger}=1\right.$, $\left.\operatorname{det}(U)=1\right)$ transforms linearly under chiral transformations,

$$
U \rightarrow U^{\prime}=L U R^{\dagger}
$$

It is most convenient to parametrize $U$ as follows

$$
U=(\sigma+i \vec{\tau} \cdot \vec{\pi}) / F, \quad \sigma^{2}+\vec{\pi}^{2}=F^{2},
$$

with $F$ the pion decay constant in the chiral limit, $F_{\pi}=F[1+\mathcal{O}(\hat{m})]=93 \mathrm{MeV}$. It is now straightforward to write down chiral covariant derivatives and construct the lowest order effective Lagrangian

$$
\begin{gathered}
\mathcal{L}_{\text {eff }}=\mathcal{L}_{\pi N}^{(1)}+\mathcal{L}_{\pi \pi}^{(2)} \\
\mathcal{L}_{\pi N}^{(1)}=\bar{\Psi}\left(i \gamma_{\mu} D^{\mu}-\stackrel{\circ}{m}+\frac{1}{2} \stackrel{\circ}{g}_{A} \gamma^{\mu} \gamma_{5} u_{\mu}\right) \Psi \\
\mathcal{L}_{\pi \pi}^{(2)}=\frac{F^{2}}{4} \operatorname{Tr}\left[\nabla_{\mu} U \nabla^{\mu} U^{\dagger}\right]+\frac{F^{2} M^{2}}{4} \operatorname{Tr}\left[U+U^{\dagger}\right],
\end{gathered}
$$

with $u_{\mu}=i u^{\dagger} \nabla_{\mu} U u^{\dagger}$. Here, the superscript 'o' denotes quantities in the chiral limit, i.e. $Q=\stackrel{\circ}{Q}[1+\mathcal{O}(\hat{m})]$ (with the exception of $M$ which is the leading term in the quark mass expansion of the pion mass and $F), g_{A}$ is the axial-vector coupling constant measured in neutron $\beta$-decay, $g_{A}=1.26$ and $m$ denotes the nucleon mass. For the three flavor case, one has of course two axial couplings. The chiral dimension (power) of the respective terms is denoted by the superscripts ' $(\mathrm{i})$ ' $(\mathrm{i}=1,2)$. The pertinent covariant derivatives are

$$
\begin{gathered}
\nabla_{\mu} U=\partial_{\mu} U-i e A_{\mu}[Q, U] \\
D_{\mu} \Psi=\partial_{\mu} \Psi+\frac{1}{2}\left\{u^{\dagger}\left(\partial_{\mu}-i e A_{\mu} Q\right) u+u\left(\partial_{\mu}-i e A_{\mu} Q\right) u^{\dagger}\right\} \Psi=\partial_{\mu} \Psi+\Gamma_{\mu} \Psi
\end{gathered}
$$


with $Q=\operatorname{diag}(1,0)$ the (nucleon) charge matrix and I only consider external vector fields, i.e. the photon $A_{\mu}$. $D_{\mu}$ transforms homogeneously under chiral transformation, $D_{\mu}^{\prime}=K D_{\mu} K^{\dagger}$, and $\Gamma_{\mu}$ is the connection [18]. To show the strength of the effective Lagrangian approach, let me quickly derive the so-called Goldberger-Treiman relation (GTR) [21]. For that, I set $A_{\mu}=0$ and expand the pion-nucleon Lagrangian to order $\vec{\pi}$,

$$
\mathcal{L}_{\pi N}^{(1)}=\bar{\Psi}\left(i \gamma_{\mu} \partial^{\mu}-\stackrel{\circ}{m}\right) \Psi-\frac{\stackrel{\circ}{A}_{A}}{F} \bar{\Psi} \gamma^{\mu} \gamma_{5} \frac{\vec{\tau}}{2} \Psi \cdot \partial_{\mu} \vec{\pi}+\ldots
$$

from which we read off the $N N \pi$ vertex in momentum space

$$
V_{N N \pi}=\frac{\stackrel{\circ}{g}_{A}}{2 F} \gamma_{\mu} q^{\mu} \gamma_{5} \vec{\tau}
$$

where the momentum $q_{\mu}$ is out-going. The transition amplitude for single pion emission off a nucleon takes the form

$$
T_{N N \pi}=-i \bar{u}\left(p^{\prime}\right) V_{N N \pi} u(p)=i \frac{\stackrel{\circ}{g}_{A}}{F} \stackrel{\circ}{m} \bar{u}\left(p^{\prime}\right) \gamma_{5} u(p) \tau^{i}
$$

where I have used the Dirac equation $\bar{u}\left(p^{\prime}\right) \gamma_{\mu} q^{\mu} \gamma_{5} u(p)=-2 \stackrel{\circ}{m} \bar{u}\left(p^{\prime}\right) \gamma_{5} u(p)$ with $q=p^{\prime}-p$. Comparing eq.(13) with the canonical form of the transition amplitude

$$
T_{N N \pi}=i \stackrel{\circ}{g}_{\pi N} \bar{u}\left(p^{\prime}\right) \gamma_{5} u(p)
$$

one arrives directly at the GTR,

$$
\stackrel{\circ}{g}_{\pi N}=\frac{\stackrel{\circ}{g}_{A} \stackrel{\circ}{m}}{F}
$$

which is fulfilled within $5 \%$ in nature. This relation is particularly intriguing because it links the strong pion-nucleon coupling constant to some weak interaction quantities like $g_{A}$ and $F_{\pi}$ as a consequence of the chiral symmetry. Finally, if one wants to discuss processes with two (or more) nucleons in the initial and final state, one has to add a string of terms of the type

$$
\mathcal{L}_{\bar{\Psi} \Psi \bar{\Psi} \Psi}+\mathcal{L}_{\bar{\Psi} \Psi \bar{\Psi} \Psi \bar{\Psi} \Psi}+\ldots
$$

which are also subject to a chiral expansion and contain LECs which can only be determined in few or many nucleon (baryon) processes.

Clearly, the appearance of the mass scale $\stackrel{\circ}{m}$ in eq.(17) causes trouble. To be precise, if one calculates the self-energy of the nucleon mass to one loop, one encounters terms of dimension zero, i.e. in dimensional regularization one finds a term of the type 15

$$
\mathcal{L}_{\pi N}^{(0)}=c_{0} \bar{\Psi} \Psi, \quad c_{0} \sim\left(\frac{\stackrel{m}{m}}{F}\right)^{2} \frac{1}{d-4}+\ldots
$$


where the ellipsis stands for terms which are finite as $d \rightarrow 4$. Such terms clearly make it difficult to organize the chiral expansion in a straightforward and simple manner. They can only be avoided if the additional mass scale $\stackrel{\circ}{m} \sim 1 \mathrm{GeV}$ can be eliminated from the lowest order effective Lagrangian. (Notice here the difference to the pion case - there the mass vanishes as the quark masses are sent to zero.) To do that, consider the mass of the nucleon large compared to the typical external momenta transferred by pions or photons and write the nucleon four-momentum as

$$
p_{\mu}=m v_{\mu}+\ell_{\mu}, \quad p^{2}=m^{2}, \quad v \cdot \ell \ll m .
$$

Notice that to this order we do not have to differentiate between $m$ and $\stackrel{\circ}{m}$ and $v_{\mu}$ is the nucleon four-velocity (in the rest-frame, we have $v_{\mu}=(1, \overrightarrow{0})$ ). In that case, we can decompose the wavefunction $\Psi$ into velocity eigenstates [16] [20]

$$
\Psi(x)=\exp [-i \stackrel{\circ}{m} v \cdot x][H(x)+h(x)]
$$

with

$$
P_{v} H=H, P_{v} h=-h, \quad P_{v}=\frac{1}{2}\left(1+\gamma^{\mu} v_{\mu}\right) .
$$

One now eliminates the 'small' component $h(x)$ either by using the equations of motion or path-integral methods. The Dirac equation for the velocity-dependent baryon field $H=H_{v}$ (I will always suppress the label ' $v$ ') takes the form $i v \cdot \partial H_{v}=0$ to lowest order in $1 / m$. This allows for a consistent chiral counting as described below and the effective pion-nucleon Lagrangian takes the form:

$$
\mathcal{L}_{\pi N}^{(1)}=\bar{H}\left(i v \cdot D+\stackrel{\circ}{g}_{A} S \cdot u\right) H+\mathcal{O}\left(\frac{1}{m}\right)
$$

with $S_{\mu}$ the covariant spin-operator

$$
S_{\mu}=\frac{i}{2} \gamma_{5} \sigma_{\mu \nu} v^{\nu}, S \cdot v=0,\left\{S_{\mu}, S_{\nu}\right\}=\frac{1}{2}\left(v_{\mu} v_{\nu}-g_{\mu \nu}\right),\left[S_{\mu}, S_{\nu}\right]=i \epsilon_{\mu \nu \gamma \delta} v^{\gamma} S^{\delta}
$$

in the convention $\epsilon^{0123}=-1$. Two important observations can be made. Eq. (21) does not contain the nucleon mass term any more and also, all Dirac matrices can be expressed as combinations of $v_{\mu}$ and $S_{\mu}$ [16,

$$
\begin{gathered}
\bar{H} \gamma_{\mu} H=v_{\mu} \bar{H} H, \bar{H} \gamma_{5} H=0, \bar{H} \gamma_{\mu} \gamma_{5} H=2 \bar{H} S_{\mu} H \\
\bar{H} \sigma_{\mu \nu} H=2 \epsilon_{\mu \nu \gamma \delta} v^{\gamma} \bar{H} S^{\delta} H, \bar{H} \gamma_{5} \sigma_{\mu \nu} H=2 i \bar{H}\left(v_{\mu} S_{\nu}-v_{\nu} S_{\mu}\right) H
\end{gathered}
$$

to leading order in $1 / m$. More precisely, this means e.g. $\bar{H} \gamma_{5} H=\mathcal{O}(1 / m)$. We read off the nucleon propagator,

$$
S_{N}(\omega)=\frac{i}{\omega+i \eta}, \quad \omega=v \cdot \ell, \quad \eta>0
$$

and the Feynman insertion for the emission a pion with momentum $\ell$ from a nucleon is

$$
\frac{g_{A}}{F_{\pi}} \tau^{a} S \cdot \ell
$$

Notice that from now on I will not always distinguish between the observables and their chiral limit values (although that distinction should be kept in mind). Before proceeding with some actual calculations in heavy baryon CHPT (HBCHPT), let me outline the chiral power counting which is used to organize the various terms in the energy expansion. 


\section{Chiral power counting}

To calculate any process to a given order, it is useful to have a compact expression for the chiral power counting [14] [12]. First, I will restrict myself to purely mesonic or single-baryon processes. Since these arguments are general, I will consider the three flavor case. Any amplitude for a given physical process has a certain chiral dimension $D$ which keeps track of the powers of external momenta and meson masses. The building blocks to calculate this chiral dimension from a general Feynman diagram in the CHPT loop expansion are (i) $I_{M}$ Goldstone boson (meson) propagators $\sim 1 /\left(\ell^{2}-M^{2}\right.$ ) (with $M=M_{\pi, K, \eta}$ the meson mass) of dimension $D=-2$, (ii) $I_{B}$ baryon propagators $\sim 1 / v \cdot \ell$ (in HBCHPT) with $D=-1$, (iii) $N_{d}^{M}$ mesonic vertices with $d=2,4,6, \ldots$ and (iv) $N_{d}^{M B}$ meson-baryon vertices with $d=1,2,3, \ldots$. Putting these together, the chiral dimension $D$ of a given amplitude reads

$$
D=4 L-2 I_{M}-I_{B}+\sum_{d} d\left(N_{d}^{M}+N_{d}^{M B}\right)
$$

with $L$ the number of loops. For connected diagrams, one can use the general topological relation

$$
L=I_{M}+I_{B}-\sum_{d}\left(N_{d}^{M}+N_{d}^{M B}\right)+1
$$

to eliminate $I_{M}$ :

$$
D=2 L+2+I_{B}+\sum_{d}(d-2) N_{d}^{M}+\sum_{d}(d-2) N_{d}^{M B} .
$$

Lorentz invariance and chiral symmetry demand $d \geq 2$ for mesonic interactions and thus the term $\sum_{d}(d-2) N_{d}^{M}$ is non-negative. Therefore, in the absence of baryon fields, Eq. (28) simplifies to 14

$$
D=2 L+2+\sum_{d}(d-2) N_{d}^{M} \geq 2 L+2 .
$$

To lowest order $p^{2}$, one has to deal with tree diagrams $(L=0)$ only. Loops are suppressed by powers of $p^{2 L}$.

Another case of interest for us has a single baryon line running through the diagram (i.e., there is exactly one baryon in the in- and one baryon in the out-state). In this case, the identity

$$
\sum_{d} N_{d}^{M B}=I_{B}+1
$$

holds leading to 12

$$
D=2 L+1+\sum_{d}(d-2) N_{d}^{M}+\sum_{d}(d-1) N_{d}^{M B} \geq 2 L+1
$$


Therefore, tree diagrams start to contribute at order $p$ and one-loop graphs at order $p^{3}$. Obviously, the relations involving baryons are only valid in HBCHPT.

Let me now consider diagrams with $N_{\gamma}$ external photons. Since gauge fields like the electromagnetic field appear in covariant derivatives, their chiral dimension is obviously $D=1$. One therefore writes the chiral dimension of a general amplitude with $N_{\gamma}$ photons as

$$
D=D_{L}+N_{\gamma}
$$

where $D_{L}$ is the degree of homogeneity of the (Feynman) amplitude $A$ as a function of external momenta $(p)$ and meson masses $(M)$ in the following sense (see also [22]):

$$
A\left(p, M ; C_{i}^{r}(\lambda), \lambda / M\right)=M^{D_{L}} A\left(p / M, 1 ; C_{i}^{r}(\lambda), \lambda / M\right),
$$

where $\lambda$ is an arbitrary renormalization scale and $C_{i}^{r}(\lambda)$ denote renormalized LECs. From now on, I will suppress the explicit dependence on the renormalization scale and on the LECs. Since the total amplitude is independent of the arbitrary scale $\lambda$, one may in particular choose $\lambda=M$. Note that $A(p, M)$ has also a certain physical dimension (which is of course independent of the number of loops and is therefore in general different from $\left.D_{L}\right)$. The correct physical dimension is ensured by appropriate factors of $F_{\pi}$ and $m$ in the denominators.

Finally, consider a process with $E_{n}\left(E_{n}=4,6, \ldots\right)$ external baryons (nucleons). The corresponding chiral dimension $D_{n}$ follows to be [23]

$$
D_{n}=2(L-C)+4-\frac{1}{2} E_{n}+\sum_{i} V_{i} \Delta_{i}, \quad \Delta_{i}=d_{i}+\frac{1}{2} n_{i}-2,
$$

where $C$ is the number of connected pieces and one has $V_{i}$ vertices of type $i$ with $d_{i}$ derivatives and $n_{i}$ baryon fields (these include the mesonic and meson-baryon vertices discussed before). Chiral symmetry demands $\Delta_{i} \geq 0$. As before, loop diagrams are suppressed by $p^{2 L}$. Notice, however, that this chiral counting only applies to the irreducible diagrams and not to the full S-matrix since reducible diagrams can lead to IR pinch singularities and need therefore a special treatment (for details, see refs. 223] [24]).

I will now briefly discuss the general structure of the effective Lagrangian based on these power counting rules, restricting myself again to the two-flavor case and processes with one nucleon in the asymptotic in- and out-states. While the lowest order Lagrangian eq.(21) has $D=1$, one can construct a string of local operators with $D=2,3,4, \ldots$.. Oneloop diagrams start at order $p^{3}$ if one only uses insertions from $\mathcal{L}_{\pi N}^{(1)}$. Two-loop graphs are suppressed by two more powers of $p$ so that within the one-loop approximation one should consider tree diagrams from

$$
\mathcal{L}_{\pi N}=\mathcal{L}_{\pi N}^{(1)}+\mathcal{L}_{\pi N}^{(2)}+\mathcal{L}_{\pi N}^{(3)}+\mathcal{L}_{\pi N}^{(4)}
$$


and loop diagrams with insertions from $\mathcal{L}_{\pi N}^{(1,2)}$. It is important to stress that not all of the terms in $\mathcal{L}_{\pi N}^{(2,3,4)}$ contain LECs, but some of the coefficients are indeed fixed for kinematical or similar reasons. I will discuss the general structure of $\mathcal{L}_{\pi N}^{(2)}$ in section 5 . It should also be stressed that although there are many terms in $\mathcal{L}_{\pi N}^{(2,3,4)}$, for a given process most of them do not contribute. As an example let me quote the order $q^{4}$ calculation of the nucleons' electromagnetic polarizabilities [25] which involves altogether four LECs from $\mathcal{L}_{\pi N}^{(2)}$ and four from $\mathcal{L}_{\pi N}^{(4)}$, a number which can certainly be controlled. To all of this, one has of course to add the purely mesonic Lagrangian $\mathcal{L}_{\pi \pi}^{(2)}+\mathcal{L}_{\pi \pi}^{(4)}$ [9] [10].

\section{A simple calculation}

In this section, I will present a typical calculation, namely the nucleon mass shift from the pion loop (in the one-loop approximation). The full result can e.g. be found in refs. 26] [20]. A similar sample calculation has been given in the lectures by Jenkins and Manohar [26], but I will use another method which is easier to generalize to processes with external photons.

Consider the Feynman diagram where the nucleon emits a pion of momentum $\ell$ and absorbs the same pion (which is incoming with momentum $-\ell$ ). Using eqs.(24,25) and the relativistic propagator for the pion, the mass shift $\delta m$ is given by

$$
\delta m=i \frac{3 g_{A}^{2}}{F_{\pi}^{2}} \int \frac{d^{d} \ell}{(2 \pi)^{d}} \frac{i}{\ell^{2}-M_{\pi}^{2}+i \eta} \frac{i}{-v \cdot \ell+i \eta} S \cdot(-\ell) S \cdot \ell,
$$

making use of $\tau^{a} \tau^{a}=3$. From the anti-commutation relation of two spin matrices, eq.(22), and by completing the square we have

$$
S_{\mu} S_{\nu} \ell^{\mu} \ell^{\nu}=\frac{1}{4}\left(v \cdot \ell v \cdot \ell+M_{\pi}^{2}-\ell^{2}-M_{\pi}^{2}\right)
$$

so that

$$
\delta m=i \frac{3 g_{A}^{2}}{4 F_{\pi}^{2}} \int \frac{d^{d} \ell}{(2 \pi)^{d}}\left[\frac{1}{v \cdot \ell-i \eta}+\frac{v \cdot \ell}{M_{\pi}^{2}-\ell^{2}-i \eta}-\frac{M_{\pi}^{2}}{\left(M_{\pi}^{2}-\ell^{2}-i \eta\right)(v \cdot \ell-i \eta)}\right] .
$$

To calculate this integral, we make use of dimensional regularization. The first term in the square brackets vanishes in dimensional regularization (see e.g. ref.[27]) and the second one is odd under $\ell \rightarrow-\ell$, i.e. it also vanishes. So we are left with

$$
\begin{gathered}
\delta m=\frac{3 g_{A}^{2}}{4 F_{\pi}^{2}} J(0) M_{\pi}^{2} \\
J(0)=\frac{1}{i} \int \frac{d^{d} \ell}{(2 \pi)^{d}} \frac{1}{\left(M_{\pi}^{2}-\ell^{2}-i \eta\right)(v \cdot \ell-i \eta)} .
\end{gathered}
$$


The remaining task is to evaluate $J(0)$. For that, we use the identity

$$
\frac{1}{A B}=\int_{0}^{\infty} d y \frac{2}{[A+2 y B]^{2}},
$$

define $\ell^{\prime}=\ell-y v$, complete the square and use $v^{2}=1$,

$$
\begin{aligned}
J(0) & =\frac{1}{i} \int_{0}^{\infty} d y \int \frac{d^{d} \ell^{\prime}}{(2 \pi)^{d}} \frac{1}{\left[M_{\pi}^{2}+y^{2}+\ell^{\prime 2}-i \eta\right]^{2}} \\
& =\frac{2}{(2 \pi)^{d}} \int_{0}^{\infty} d y \int_{0}^{\infty} d \ell^{\prime} \frac{\left(\ell^{\prime}\right)^{d-1}}{\left[M_{\pi}^{2}+y^{2}+\ell^{\prime 2}\right]^{2}} \frac{2 \pi^{d / 2}}{\Gamma(d / 2)},
\end{aligned}
$$

where we have performed a Wick rotation, $\ell_{0} \rightarrow i \ell_{0}$ and dropped the $i \eta$. The last factor in eq.(43) is the surface of the sphere in $d$ dimensions. Introducing polar coordinates, $y=r \cos \phi$ and $\ell^{\prime}=r \sin \phi$ and noting that the Jacobian of this transformation is $r$, we have

$$
J(0)=\frac{4(4 \pi)^{-d / 2}}{\Gamma(d / 2)} \int_{0}^{\infty} d r \frac{r^{d}}{\left(r^{2}+M_{\pi}^{2}\right)^{2}} \int_{0}^{\pi / 2} d \phi(\sin \phi)^{d-1} .
$$

We then perform the further substitution $r=M_{\pi} \tan \Phi$ in the $r$-integral. Then, both integrals appearing in eq.(44) can be expressed in terms of products of $\Gamma$ functions with the result

$$
J(0)=M_{\pi}^{d-3}(4 \pi)^{-d / 2} \Gamma\left(\frac{1}{2}\right) \Gamma\left(\frac{3-d}{2}\right)=-\frac{M_{\pi}}{8 \pi},
$$

where in the last step I have set $d=4$. This leads us to

$$
\delta m=i \Sigma=-\frac{3 g_{A}^{2}}{32 \pi} \frac{M_{\pi}^{3}}{F_{\pi}^{2}} .
$$

The pion loop leads to a self-energy $\Sigma$ which shifts the pole of the nucleon propagator by $\delta m$, i.e.

$$
\frac{i}{v \cdot \ell-i \Sigma}=\frac{i}{v \cdot \ell}\left(1-\Sigma \frac{i}{v \cdot \ell}\right)^{-1}=\frac{i}{v \cdot \ell}+\frac{i}{v \cdot \ell} \Sigma \frac{i}{v \cdot \ell}+\ldots=\frac{i}{v \cdot \ell-\delta m} .
$$

There are a few important remarks concerning eq.(46). First, the pion loop contribution is non-analytic in the quark masses [28]

$$
\delta m \sim(\hat{m})^{3 / 2},
$$

since

$$
M_{\pi}^{2}=B \hat{m}\left[1+\mathcal{O}\left(m_{\text {quark }}\right)\right] .
$$

The constant $B$ is related to the scalar quark condensate and is assumed to be nonvanishing in the chiral limit (supported by lattice data). (For a different scenario, see e.g. refs. [29]). Second, the pion cloud contribution is attractive, i.e. it lowers the nucleon mass, 
and third, it vanishes in the chiral limit, i.e it has the expected chiral dimension of three (since it is a one-loop graph with insertions from the lowest order effective Lagrangian). More detailed studies of the baryon masses and $\sigma$-terms can be found in refs. 30] 26] [31] 32] 33] 34 [35] [36]. Here, I only wish to state that at present it is not known whether the scalar three-flavour sector (i.e. the baryon masses and the $\sigma$-terms) can be described consistently within CHPT. It seems that one at least should go to order $q^{4}$ and it is unclear how the close-by spin-3/2 decuplet has to be treated (i.e. as effective degrees of freedom or frozen to supply large contributions to certain LECs). The problem is that the kaon loop corrections are rather large (since $\left(M_{K} / M_{\pi}\right)^{3} \sim 46$ ) and the corresponding LECs have therefore large coefficients to compensate for the meson cloud contribution. It might, however, well be that the corrections beyond leading order are much smaller. This can only be decided upon a complete calculation to order $q^{4}$.

\section{The structure of $\mathcal{L}_{\pi N}^{(2)}$}

In this section, I will first write down the order $p^{2}$ effective Lagrangian, $\mathcal{L}_{\pi N}^{(2)}$, and then discuss some of its peculiarities. Allowing for the moment for $m_{u} \neq m_{d}$, its most general form is (I only consider external scalar and vector fields, the generalization to pseudoscalar and axial-vector ones is straightforward):

$$
\begin{aligned}
& \mathcal{L}_{\pi N}^{(2)}=\bar{H}\left\{\frac{1}{2 \stackrel{\circ}{m}}(v \cdot D)^{2}-\frac{1}{2 \stackrel{\circ}{m}} D^{2}-\frac{i \stackrel{\circ}{g}_{A}}{2 \stackrel{\circ}{m}}\{S \cdot D, v \cdot u\}+c_{1} \operatorname{Tr} \chi_{+}+\left(c_{2}-\frac{\stackrel{\circ}{g}_{A}}{8 \stackrel{\circ}{m}}\right)(v \cdot u)^{2}\right. \\
& \left.\quad+c_{3} u \cdot u+\left(c_{4}+\frac{1}{4 \stackrel{\circ}{m}}\right)\left[S^{\mu}, S^{\nu}\right] u_{\mu} u_{\nu}+c_{5} \tilde{\chi}_{+}-\frac{i\left[S^{\mu}, S^{\nu}\right]}{4 \stackrel{\circ}{m}}\left(\left(1+c_{6}\right) f_{\mu \nu}^{+}+c_{7} \operatorname{Tr} f_{\mu \nu}^{+}\right)\right\} H
\end{aligned}
$$

with

$$
\chi_{ \pm}=u^{\dagger} \chi u^{\dagger} \pm u \chi^{\dagger} u, \quad \tilde{\chi}_{+}=\chi_{+}-\frac{1}{2} \operatorname{Tr}\left(\chi_{+}\right), \quad f_{\mu \nu}^{+}=u^{\dagger} F_{\mu \nu} u+u F_{\mu \nu} u^{\dagger}
$$

Here, $\chi=2 B \mathcal{M}\left(\mathcal{M}\right.$ is the quark mass matrix) and $F_{\mu \nu}=\partial_{\mu} A_{\nu}-\partial_{\nu} A_{\mu}$ the canonical photon field strength tensor. The term proportional to $c_{5}$ vanishes in the isospin limit $m_{u}=m_{d}$. One observes that some of the terms in eq.(50) have no LECs but rather fixed coefficients. The origin of this is clear, these terms stem from the expansion of the relativistic $\pi N$ Lagrangian. To see this in more detail, use the equation of motion for the small component field $h(x)$,

$$
h=\frac{1}{2}(1-\psi) \frac{1}{2 \stackrel{\circ}{m}}\left(i \not D+\frac{\stackrel{\circ}{A}_{A}}{2} \not \gamma_{5}\right) H+\mathcal{O}\left(1 / \stackrel{\circ}{m}^{2}\right)
$$


to construct

$$
\mathcal{L}_{\pi N}^{(2)}=\frac{1}{2 \stackrel{\circ}{m}} \bar{H}\left(i \not D+\frac{\stackrel{\circ}{g}_{A}}{2} \not \gamma_{5}\right) \frac{1-\psi}{2} \bar{H}\left(i \not D+\frac{\stackrel{\circ}{g}_{A}}{2} \not \gamma_{5}\right) H .
$$

Altogther, we have four different products of terms in eq.(53). Let me consider the one proportional to $\not D \not D$, the other contributions can be calculated in a similar fashion:

$$
\frac{i^{2}}{2 \stackrel{\circ}{m}} \bar{H} \not D \frac{1-\psi}{2} \not D H=-\frac{1}{2 \stackrel{\circ}{m}} D^{\mu} D^{\nu} \bar{H}\left[\gamma_{\mu} \frac{1-\psi}{2} \gamma_{\nu}\right] H .
$$

Straightforward application of the $\gamma$-matrix algebra allows us to write the term in the square brackets on the r.h.s. as

$$
\bar{H}[\ldots] H=\bar{H}\left[g_{\mu \nu}-i \sigma_{\mu \nu}-\gamma_{\mu} v_{\nu}\right] H,
$$

where I have used that $P_{v} H=H$. Collecting pieces, we have

$$
\frac{i^{2}}{2 \stackrel{\circ}{m}} \bar{H}\left\{g_{\mu \nu} D^{\mu} D^{\nu}-2 i \epsilon_{\mu \nu \alpha \beta} D^{\mu} D^{\nu} v^{\alpha} S^{\beta}-v_{\mu} D^{\mu} v_{\nu} D^{\nu}\right\} H .
$$

This simplifies further since $\epsilon_{\mu \nu \alpha \beta}$ and $D^{\mu} D^{\nu}$ are anti- and symmetric under $\mu \leftrightarrow \nu$, respectively,

$$
\frac{1}{2 \stackrel{\circ}{m}} \bar{H}\left\{-D^{2}+(v \cdot D)^{2}+i \epsilon_{\mu \nu \alpha \beta}\left[D^{\mu}, D^{\nu}\right] v^{\alpha} S^{\beta}\right\} H .
$$

Finally, the commutator of two chiral covariant derivatives is related to the chiral connection $\Gamma_{\mu}$ via

$$
\left[D_{\mu}, D_{\nu}\right]=\partial_{\mu} \Gamma_{\nu}-\partial_{\nu} \Gamma_{\mu}+\left[\Gamma_{\mu}, \Gamma_{\nu}\right] .
$$

We can work this out for the explicit form of the connection given in eq.(10) and find

$$
\left[D_{\mu}, D_{\nu}\right]=-\frac{i}{2} f_{\mu \nu}^{+}+\frac{1}{4}\left[u_{\mu}, u_{\nu}\right] .
$$

Putting everything together, we find that the first three terms in eq.(50) plus the piece proportional to $\stackrel{\circ}{g}_{A}^{2} /(8 \stackrel{\circ}{m})$ in the fifth and the piece proportional to $1 /(4 \stackrel{\circ}{m})$ in the seventh term are generated by expanding the operator $\not D \not D$. The first two contributions are corrections to the kinetic energy and contain (besides others) a two-photon-nucleon seagull which leads to the correct LET for low-energy Compton scattering (see section 6). Similarly, the third term has no free coefficient since it gives the leading term in the quark mass expansion of the electric dipole amplitude $E_{0+}$ in neutral pion photoproduction off protons, $E_{0+} \sim M_{\pi} / m$. These terms have no direct relativistic counter parts but are simply due to the expansion of the relativistic pion-nucleon effective Lagrangian. Such constraints were e.g. not accounted for in eq.(17) of ref. [16]. The finite coefficients $c_{1,2,3,4}$ can be determined from the pion-nucleon $\sigma$-term and $\mathrm{S}$ - and $\mathrm{P}$-wave $\pi N$ scattering lengths [20] [37 [38]. The last two terms in eq.(50) are easy to pin down, they are related 
to the isoscalar and isovector anomalous magnetic moment of the nucleon (in the chiral limit) [20]

$$
c_{6}=\stackrel{\circ}{\kappa}_{V} \quad c_{7}=\frac{1}{2}\left(\stackrel{\circ}{\kappa}_{S}-\stackrel{\circ}{\kappa}_{V}\right)
$$

Apart from the LEC $c_{5}$, all of these coefficients have been determined. For a meaningful comparison, I normalize all terms to $(1 / 2 \stackrel{\circ}{m})$, i.e. I define

$$
c_{i}^{\prime}=2 \stackrel{\circ}{m} c_{i}(i=1,2,3,4), \quad c_{6,7}^{\prime}=2 c_{6,7},
$$

and use $\stackrel{\circ}{m}=m=\left(m_{p}+m_{n}\right) / 2=0.93892 \mathrm{GeV}$ (which is correct to this order). The resulting values are summarized in table 1 .

\begin{tabular}{|crll|}
\hline LEC & \multicolumn{1}{c}{ Value } & Source & Ref. \\
\hline$c_{1}^{\prime}$ & $-1.63 \pm 0.21$ & $\sigma_{\pi N}(0)$ & 20 \\
$c_{2}^{\prime}$ & $6.20 \pm 0.38$ & $\pi N \rightarrow \pi N$ & 37 \\
$c_{3}^{\prime}$ & $-9.86 \pm 0.41$ & $\pi N \rightarrow \pi N$ & 38 \\
$c_{4}^{\prime}$ & $7.73 \pm 0.18$ & $\pi N \rightarrow \pi N$ & 38 \\
$c_{6}^{\prime}$ & $7.41 \pm 0.00$ & $\kappa_{p, n}$ & \\
$c_{7}^{\prime}$ & $-7.17 \pm 0.00$ & $\kappa_{p, n}$ & 20 \\
\hline
\end{tabular}

Table 1: Numerical values of the LECs in $\mathcal{L}_{\pi N}^{(2)}$ (with the exception of $c_{5}$ ).

I would like to stress that the coefficients $c_{1}$ and $c_{2}$ have been determined from the chiral corrections to the $\pi N \sigma$-term and to the S-wave scattering length $a^{+}$. Both of the quantities are either subject to large higher order corrections or are not very accurately determined empirically. For the LEC $c_{3}^{\prime}$, there is another way of fixing it. The value given in the table stems from the so-called axial polarizability $\alpha_{A}$ and the error solely reflects the uncertainty in the empirical value of $\alpha_{A}$. One can also use the pion-nucleon $\mathrm{P}$-wave scattering volume $P_{1}^{+} \sim\left(4 a_{33}+2 a_{31}+2 a_{13}+a_{11}\right)$ (with $a_{2 I, 2 J}$ the scattering volumes in the respective channels) to get this LEC. One finds $c_{3}^{\prime}=-11.87 \pm 2.69$, which is somewhat larger than the value given in table 1 but overlaps within one standard deviation. If one is conservative, one would therefore estimate the theoretical error to be \pm 1.5 for $c_{i}^{\prime}$ $(i=1,2,3,4)$. For $c_{6}^{\prime}$ and $c_{7}^{\prime}$, this theoretical uncertainty is clearly much smaller since the anomalous magnetic moments of the proton and the neutron are well known. In any case, it would be very useful to have another and possibly more accurate determination of these LECs. In fact, van Kolck and collaborators [39] have considered the chiral expansion of the nucleon-nucleon interaction to one-loop accuracy. Naturally, they also have some of the terms appearing in eq.(50) and they call the corresponding LECs $B_{1,2,3}$. Translating their language into the one used here, one has

$$
B_{1}=4 c_{3}, \quad B_{2}=8 c_{1}, \quad B_{3}=-4 c_{4}-\frac{1}{\stackrel{\circ}{m}} .
$$


In a best fit to the deuteron properties and the NN phase shifts at low energies these parameters were left completely unconstrained. The resulting values are $B_{1}=3.42$, $B_{2}=8.55$ and $B_{3}=17.7$ [40] which translates into $c_{1}^{\prime}=2.01, c_{3}^{\prime}=1.60$ and $c_{4}^{\prime}=-8.80$ (all in $\mathrm{GeV}^{-1}$ ). The value for $c_{2}^{\prime}$ is considerably smaller than the one given in table 1 whereas $c_{1}^{\prime}$ and $c_{4}^{\prime}$ are of comparable magnitude but have the opposite sign to the numbers given in table 1 . To my opinion, this discrepancy is rooted in the fact that the fit contains a rather large number of LECs (mostly related to four-nucleon contact terms) so that the individual ones like $B_{1,2,3}$ are not very precisely determined (lowest order one-pion exchange and some of the contact terms are most important to give the gross features of the NN interaction, so that a term like the one $\sim B_{3}$, which is the $\sigma$-term, does not carry too much statistical weight). In fact, one should rather use the values given from $\pi N$ scattering as input in the much tougher problem of the nuclear forces. Naturally, one can ask the question whether one can understand the values of the $c_{i}^{\prime}$ given in table 1 ? This is indeed the case as detailed in ref. 38]. To be specific, let me pick $c_{3}^{\prime}$. Consider an EFT of meson resonances $(\mathrm{M}=\mathrm{V}, \mathrm{A}, \mathrm{S}, \mathrm{P})$ chirally coupled to the Goldstone bosons and matter fields $(N)$ as well as baryonic excitations $\left(N^{\star}\right)$. Integrating out the meson and nucleon resonances,

$$
\int[d M]\left[d N^{\star}\right] \exp i \int d x \tilde{\mathcal{L}}_{\mathrm{eff}}\left[U, M, N, N^{\star}\right]=\exp i \int d x \mathcal{L}_{\mathrm{eff}}[U, N],
$$

one is left with a string of higher dimensional operators $(D=2,3, \ldots)$ contributing to the effective pion-nucleon Lagrangian in a manifestly chirally invariant manner and with coefficients given entirely in terms of resonance masses and coupling constants of the resonance fields to the Goldstone bosons and nucleons. In the meson sector, this works remarkably well 41. Here, it has more the status of a working hypothesis (but a wellfounded one). For the LEC $c_{3}^{\prime}$, we have $\Delta, N^{\star}(1440)$ and scalar meson exchange. Varying the corresponding couplings within their allowed values, one finds [42] [38]

$$
\begin{gathered}
c_{3, \mathrm{res}}^{\prime}=c_{3, \Delta}^{\prime}+c_{3, N^{\star}}^{\prime}+c_{3, S}^{\prime} \\
=(-4.7 \ldots-6.0)+(-0.2 \ldots-0.4)+(-1.9 \ldots-3.0) \\
=-6.8 \ldots-9.4 .
\end{gathered}
$$

This number is of comparable size to the one given in table 1, although the resulting values tend to come out on the lower side. Nevertheless, such considerations seem to give credit to the resonance saturation picture of the LECs also in the baryon sector. It is worth to point out that besides a large contribution from the $\Delta$, scalar meson exchange gives a sizeable chunk to $c_{3, \text { res }}^{\prime}$. This shows that the resonance exchange picture is more complex in the baryon sector than in the mesonic one. More work in this direction is clearly needed. 
The important lesson to be learned from this discussion is that in HBCHPT we find in $\mathcal{L}_{\pi N}^{(2,3, \ldots)}$ terms which have no LECs but rather coefficients which are fixed. This is an artefact of the dual expansion in small momenta $p$ versus the chiral symmetry breaking scale and versus inverse powers of the nucleon mass, i.e.

$$
\frac{p}{\Lambda_{\chi}}, \frac{p}{m} .
$$

In practice, since $m \sim \Lambda_{\chi}$, one has essentially one expansion parameter besides the one due to the effect of the finite quark masses. Since loops only appear at $D=3$, all the LECs in $\mathcal{L}_{\pi N}^{(2)}$ are finite (as already should have become clear from the previous discussion). At the next order, $p^{3}$, divergences appear. Ecker [43] has recently calculated the full determinant using heat-kernel methods and given the divergent terms,

$$
\mathcal{L}_{\pi N}^{(3)}=\frac{1}{(4 \pi)^{2}} \sum_{i=1}^{22} b_{i} \bar{H}(x) O_{i}(x) H(x)
$$

with

$$
b_{i}=b_{i}^{r}(\lambda)+\Gamma_{i} L(\lambda), \quad L \sim \frac{1}{d-4} .
$$

The $O_{i}$ are monomials in the fields and have dimension three. Their explicit forms together with the values of the $\Gamma_{i}$ can be found in ref.43. Only a few of the finite $b_{i}^{r}$ have either been determined from phenomenology or estimated from resonance exchange [31] [20] [37] [44. For $\mathcal{L}_{\pi N}^{(4)}$, no systematic study exists so far but some dimension four operators have been used and their corresponding LECs fixed [25] 444].

\section{The meaning of low-energy theorems (LETs)}

In this section, I will briefly discuss the meaning of the so-called low-energy theorems. More details are given in ref. 45]. Let us first consider a well-known example of a LET involving the electromagnetic current. Consider the scattering of very soft photons on the proton, i.e., the Compton scattering process $\gamma\left(k_{1}\right)+p\left(p_{1}\right) \rightarrow \gamma\left(k_{2}\right)+p\left(p_{2}\right)$ and denote by $\varepsilon\left(\varepsilon^{\prime}\right)$ the polarization vector of the incoming (outgoing) photon. The transition matrix element $T$ (normalized to $d \sigma / d \Omega=|T|^{2}$ ) can be expanded in a Taylor series in the small parameter $\delta=\left|\overrightarrow{k_{1}}\right| / m$. In the forward direction and in a gauge where the polarization vectors have only space components, $T$ takes the form

$$
T=c_{0} \vec{\varepsilon}^{\prime} \cdot \vec{\varepsilon}+i c_{1} \delta \vec{\sigma} \cdot\left(\vec{\varepsilon}^{\prime} \times \vec{\varepsilon}\right)+\mathcal{O}\left(\delta^{2}\right)
$$

The parameter $\delta$ can be made arbitrarily small in the laboratory so that the first two terms in the Taylor expansion (68) dominate. To be precise, the first one proportional to $c_{0}$ gives the low-energy limit for the spin-averaged Compton amplitude, while the second $\left(\sim c_{1}\right)$ is of pure spin-flip type and can directly be detected in polarized photon proton 
scattering (to my knowledge, such a test has not yet been performed). The pertinent LETs fix the values of $c_{0}$ and $c_{1}$ in terms of measurable quantities 46,

$$
c_{0}=-\frac{Z^{2} e^{2}}{4 \pi m}, \quad c_{1}=-\frac{Z^{2} e^{2} \kappa_{p}^{2}}{8 \pi m}
$$

with $Z=1$ the charge of the proton. To arrive at Eq. (69), one only makes use of gauge invariance and the fact that the $T$-matrix can be written in terms of a timeordered product of two conserved vector currents sandwiched between proton states. The derivation proceeds by showing that for small enough photon energies the matrix element is determined by the electromagnetic form factor of the proton at $q^{2}=0$ 46].

Similar methods can be applied to other than the electromagnetic currents. In strong interaction physics, a special role is played by the axial-vector currents. The associated symmetries are spontaneously broken giving rise to the Goldstone matrix elements

$$
\left\langle 0\left|A_{\mu}^{a}(0)\right| \pi^{b}(p)\right\rangle=i \delta^{a b} F_{\pi} p_{\mu}
$$

where $a, b$ are isospin indices. In the chiral limit, the massless pions play a similar role as the photon and many LETs have been derived for "soft pions". In light of the previous discussion on Compton scattering, the most obvious one is Weinberg's prediction for elastic $\pi p$ scattering [47]. We only need the following translations :

$$
\begin{gathered}
<p\left|T j_{\mu}^{\mathrm{em}}(x) j_{\nu}^{\mathrm{em}}(0)\right| p>\rightarrow<p\left|T A_{\mu}^{\pi^{+}}(x) A_{\nu}^{\pi^{-}}(0)\right| p>, \\
\partial^{\mu} j_{\mu}^{\mathrm{em}}=0 \rightarrow \partial^{\mu} A_{\mu}^{\pi^{-}}=0 .
\end{gathered}
$$

In contrast to photons, pions are not massless in the real world. It is therefore interesting to find out how the LETs for soft pions are modified in the presence of non-zero pion masses (due to non-vanishing quark masses). In the old days of current algebra, a lot of emphasis was put on the PCAC (Partial Conservation of the Axial-Vector Current) relation, consistent with the Goldstone matrix element (70),

$$
\partial^{\mu} A_{\mu}^{a}=M_{\pi}^{2} F_{\pi} \pi^{a}
$$

Although the precise meaning of (73) has long been understood [13, it does not offer a systematic method to calculate higher orders in the momentum and mass expansion of LETs. The derivation of non-leading terms in the days of current algebra and PCAC was more an art than a science, often involving dangerous procedures like off-shell extrapolations of amplitudes. In the modern language, i.e. the EFT of the Standard Model, these higher order corrections can be calculated unambigously and one correspondingly defines a low-energy theorem via: 


\section{$\mathbf{L}(\mathrm{OW}) \quad \mathbf{E}$ (NERGY) $\quad \mathbf{T}$ (HEOREM) $\quad$ OF $\quad \mathcal{O}\left(p^{n}\right)$ $\equiv$ GENERAL PREDICTION OF $\quad$ CHPT $\quad$ TO $\quad \mathcal{O}\left(p^{n}\right)$.}

By general prediction I mean a strict consequence of the SM depending on some LECs like $F_{\pi}, m, g_{A}, \kappa_{p}, \ldots$, but without any model assumption for these parameters. This definition contains a precise prescription how to obtain higher-order corrections to leading-order LETs.

The soft-photon theorems, e.g., for Compton scattering [46], involve the limit of small photon momenta, with all other momenta remaining fixed. Therefore, they hold to all orders in the non-photonic momenta and masses. In the low-energy expansion of CHPT, on the other hand, the ratios of all small momenta and pseudoscalar meson masses are held fixed. Of course, the soft-photon theorems are also valid in CHPT as in any gauge invariant quantum field theory. To understand this difference of low-energy limits, I will now rederive and extend the LET for spin-averaged nucleon Compton scattering in the framework of HBCHPT [20]. Consider the spin-averaged Compton amplitude in forward direction (in the Coulomb gauge $\varepsilon \cdot v=0$ )

$$
e^{2} \varepsilon^{\mu} \varepsilon^{\nu} \frac{1}{4} \operatorname{Tr}\left[\left(1+\gamma_{\lambda} v^{\lambda}\right) T_{\mu \nu}(v, k)\right]=e^{2}\left[\varepsilon^{2} U(\omega)+(\varepsilon \cdot k)^{2} V(\omega)\right]
$$

with $\omega=v \cdot k$ ( $k$ is the photon momentum) and

$$
T_{\mu \nu}(v, k)=\int d^{4} k \mathrm{e}^{i k \cdot x}<N(v)\left|T j_{\mu}^{\mathrm{em}}(x) j_{\nu}^{\mathrm{em}}(0)\right| N(v)>.
$$

All dynamical information is contained in the functions $U(\omega)$ and $V(\omega)$. We only consider $U(\omega)$ here and refer to Ref. 20] for the calculation of both $U(\omega)$ and $V(\omega)$. In the Thomson limit, only $U(0)$ contributes to the amplitude. In the forward direction, the only quantities with non-zero chiral dimension are $\omega$ and $M_{\pi}$. In order to make this dependence explicit, we write $U\left(\omega, M_{\pi}\right)$ instead of $U(\omega)$. With $N_{\gamma}=2$ external photons, the degree of homogeneity $D_{L}$ for a given CHPT contribution to $U\left(\omega, M_{\pi}\right)$ follows from Eq. (31) :

$$
D_{L}=2 L-1+\sum_{d}(d-2) N_{d}^{M}+\sum_{d}(d-1) N_{d}^{M B} \geq-1 .
$$

Therefore, the chiral expansion of $U\left(\omega, M_{\pi}\right)$ takes the following general form :

$$
U\left(\omega, M_{\pi}\right)=\sum_{D_{L} \geq-1} \omega^{D_{L}} f_{D_{L}}\left(\omega / M_{\pi}\right) .
$$

The following arguments illuminate the difference and the interplay between the softphoton limit and the low-energy expansion of CHPT. Let us consider first the leading terms in the chiral expansion (78) :

$$
U\left(\omega, M_{\pi}\right)=\frac{1}{\omega} f_{-1}\left(\omega / M_{\pi}\right)+f_{0}\left(\omega / M_{\pi}\right)+\mathcal{O}\left(p^{3}\right) .
$$


Eq. (77) tells us that only tree diagrams can contribute to the first two terms. However, the relevant tree diagrams do not contain pion lines. Consequently, the functions $f_{-1}$, $f_{0}$ cannot depend on $M_{\pi}$ and are therefore constants. Since the soft-photon theorem [46] requires $U\left(0, M_{\pi}\right)$ to be finite, $f_{-1}$ must actually vanish and the chiral expansion of $U\left(\omega, M_{\pi}\right)$ can be written as

$$
U\left(\omega, M_{\pi}\right)=f_{0}+\sum_{D_{L} \geq 1} \omega^{D_{L}} f_{D_{L}}\left(\omega / M_{\pi}\right) .
$$

But the soft-photon theorem yields additional information : since the Compton amplitude is independent of $M_{\pi}$ in the Thomson limit and since there is no term linear in $\omega$ in the spin-averaged amplitude, we find

$$
\lim _{\omega \rightarrow 0} \omega^{n-1} f_{n}\left(\omega / M_{\pi}\right)=0 \quad(n \geq 1)
$$

implying in particular that the constant $f_{0}$ describes the Thomson limit :

$$
U\left(0, M_{\pi}\right)=f_{0} .
$$

Let me now verify these results by explicit calculation. In the Coulomb gauge, there is no direct photon-nucleon coupling from the lowest-order effective Lagrangian $\mathcal{L}_{\pi N}^{(1)}$ since it is proportional to $\varepsilon \cdot v$. Consequently, the corresponding Born diagrams vanish so that indeed $f_{-1}=0$. On the other hand, I had argued in section 5 that the heavy mass expansion of the relativistic $\pi N$ Dirac Lagrangian leads to a Feynman insertion of the form (from the first two terms in eq.(50)):

$$
i \frac{e^{2}}{m} \frac{1}{2}\left(1+\tau_{3}\right)\left[\varepsilon^{2}-(\varepsilon \cdot v)^{2}\right]=i \frac{e^{2} Z^{2}}{m} \varepsilon^{2}
$$

producing the desired result $f_{0}=Z^{2} / m$, the Thomson limit.

At the next order in the chiral expansion, $\mathcal{O}\left(p^{3}\right)\left(D_{L}=1\right)$, the function $f_{1}\left(\omega / M_{\pi}\right)$ is given by the finite sum of 9 one-loop diagrams [48] [20]. According to Eq. [81], $f_{1}$ vanishes for $\omega \rightarrow 0$. The term linear in $\omega / M_{\pi}$ yields the leading contribution to the sum of the electric and magnetic polarizabilities of the nucleon, defined by the second-order Taylor coefficient in the expansion of $U\left(\omega, M_{\pi}\right)$ in $\omega$ :

$$
f_{1}\left(\omega / M_{\pi}\right)=-\frac{11 g_{A}^{2} \omega}{192 \pi F_{\pi}^{2} M_{\pi}}+\mathcal{O}\left(\omega^{2}\right) .
$$

The $1 / M_{\pi}$ behaviour should not come as a surprise - in the chiral limit the pion cloud becomes long-ranged (instead of being Yukawa-suppressed) so that the polarizabilities explode. This behaviour is specific to the leading contribution of $\mathcal{O}\left(p^{3}\right)$. In fact, from the general form (80) one immediately derives that the contribution of $\mathcal{O}\left(p^{n}\right)\left(D_{L}=n-2\right)$ to the polarizabilities is of the form $c_{n} M_{\pi}^{n-4}(n \geq 3)$, where $c_{n}$ is a constant that may 
be zero. One can perform a similar analysis for the amplitude $V(\omega)$ and for the spin-flip amplitude. We do not discuss these amplitudes here but refer the reader to Ref. [20] for details.

Next, let us consider the processes $\gamma N \rightarrow \pi^{0} N(N=p, n)$ at threshold, i.e., for vanishing three-momentum of the pion in the nucleon rest frame. At threshold, only the electric dipole amplitude $E_{0+}$ survives and the only quantity with non-zero chiral dimension is $M_{\pi}$. In the usual conventions, $E_{0+}$ has physical dimension -1 and it can therefore be written as

$$
E_{0+}=\frac{e g_{A}}{F} A\left(\frac{M_{\pi}}{m}, \frac{M_{\pi}}{F}\right) .
$$

The dimensionless amplitude $A$ will be expressed as a power series in $M_{\pi}$. The various parts are characterized by the degree of homogeneity (in $M_{\pi}$ ) $D_{L}$ according to the chiral expansion. Since $N_{\gamma}=1$ in the present case, we obtain from Eq. (31)

$$
D_{L}=D-1=2 L+\sum_{d}(d-2) N_{d}^{M}+\sum_{d}(d-1) N_{d}^{M B} .
$$

For the LET of $\mathcal{O}\left(p^{3}\right)$ in question, only lowest-order mesonic vertices $(d=2)$ will appear. Therefore, in this case the general formula for $D_{L}$ takes the simpler form

$$
D_{L}=2 L+\sum_{d}(d-1) N_{d}^{M B} .
$$

I will not discuss the chiral expansion of $E_{0+}$ step by step, referring to the literature 499 [50] [20] for the actual calculation and for more details to the Comment 445. Up-toand-including order $\mu^{2}$, one has to consider contributions with $D_{L}=0,1$ and 2. In fact, for neutral pion photoproduction, there is no term with $D_{L}=0$ since the time-honored Kroll-Ruderman contact term [51] where both the pion and the photon emanate from the same vertex, only exists for charged pions. For $D_{L}=2$ there is a one-loop contribution $(L=1)$ with leading-order vertices only $\left(N_{d}^{M B}=0(d>1)\right)$. It is considerably easier to work out the relevant diagrams in HBCHPT [20] than in the original derivation [49] [50]. In fact, at threshold only the so-called triangle diagram (and its crossed partner) survive out of some 60 diagrams. The main reason for the enormous simplification in HBCHPT is that one can choose a gauge without a direct $\gamma N N$ coupling of lowest order and that there is no direct coupling of the produced $\pi^{0}$ to the nucleon at threshold. Notice that the loop contributions are finite and they are identical for proton and neutron. They were omitted in the original version of the LET [52 [53] and in many later rederivations. The full LETs of $\mathcal{O}\left(p^{3}\right)$ are given by 49]

$$
\begin{gathered}
E_{0+}\left(\pi^{0} p\right)=-\frac{e g_{A}}{8 \pi F_{\pi}}\left[\frac{M_{\pi}}{m}-\frac{M_{\pi}^{2}}{2 m^{2}}\left(3+\kappa_{p}\right)-\frac{M_{\pi}^{2}}{16 F_{\pi}^{2}}+\mathcal{O}\left(M_{\pi}^{3}\right)\right] \\
E_{0+}\left(\pi^{0} n\right)=-\frac{e g_{A}}{8 \pi F_{\pi}}\left[\frac{M_{\pi}^{2}}{2 m^{2}} \kappa_{n}-\frac{M_{\pi}^{2}}{16 F_{\pi}^{2}}+\mathcal{O}\left(M_{\pi}^{3}\right)\right]
\end{gathered}
$$


We note that the electric dipole amplitude for neutral pion production vanishes in the chiral limit. By now, even the terms of order $M_{\pi}^{3}$ have been worked out, see ref. 44.

The derivation of LETs sketched above is based on a well-defined quantum field theory where each step can be checked explicitly. Nevertheless, the corrected LETs have been questioned by several authors. We find it instructive to discuss some of the arguments and assumptions that have been used to derive or rederive the original LETs. (a) Analyticity assumption: The original derivations [52] [53] and some later rederivations [55] [56] [57] [54] used a Taylor expansion of amplitudes in the variables $\nu, \nu_{B}$ (linear combinations of the usual Mandelstam variables $s$ and $u$ ). The seemingly plausible assumption that the coefficients of this expansion are analytic in $M_{\pi}$ leads directly to the original lowenergy guess (LEG). In fact, in Ref. [52] it was explicitly spelled out that this is a necessary assumption for the LEG to hold. However, as shown in Ref. [49], this assumption does not hold in QCD. Due to the Goldstone nature of the pion, some Taylor coefficients diverge in the chiral limit. This happens precisely in the loop contributions $\left(D_{L}=2\right)$ which generate infrared divergences in some coefficients. The threshold amplitude itself is perfectly wellbehaved in the chiral limit. (b) External versus internal pion mass: It has been suggested [56] [58] [59] that there is a basic difference between the external, kinematical pion mass $M_{\pi}$ and the internal mass $\bar{M}_{\pi}$ appearing in the pion propagators in loop diagrams. The assumption is that $\bar{M}_{\pi}$ appears only in relations between unrenormalized and renormalized quantities. Therefore, expressing everything in measurable, renormalized quantities, no trace of $\bar{M}_{\pi}$ is left and one recovers the original LEG since the loop contribution is to be dropped by assumption. In HBCHPT the difference between the chiral limit values $F, \stackrel{\circ}{g}_{A}$ , $M, \stackrel{\circ}{\kappa}_{p}$ and the physical parameters $F_{\pi}, g_{A}, M_{\pi}, \kappa_{p}$ only affects terms of $\mathcal{O}\left(M_{\pi}^{3}\right)$ in the LETs for $\pi^{0}$ photoproduction at threshold. Thus, the loop contribution to the LETs cannot be a renormalization effect. There is a more fundamental objection to the distinction between external and internal pion masses. QCD does not offer a consistent procedure for $M_{\pi} \rightarrow 0$ with $\bar{M}_{\pi}$ remaining finite. The only tunable mass parameters in QCD are the quark masses. Letting the quark masses tend to zero makes all pion masses vanish, whether they be external or internal. (c) Off-shell expansion: The inadmissible distinction between external and internal pion masses can also appear in an off-shell extrapolation of the amplitude. Davidson has contrasted the expansion in $M_{\pi}$ with a so-called $\omega$ expansion [60]. Keeping $M_{\pi}$ fixed, he sets the three-momentum $\vec{p}_{\pi}=0$ and expands in the pion energy $E_{\pi}=\omega$. Obviously, for $\omega \neq M_{\pi}$ this implies an off-shell extrapolation of the scattering amplitude. If one expands the amplitude first to $\mathcal{O}\left(\omega^{2}\right)$, the coefficients still depend on $M_{\pi}$. Expanding those coefficients in a second step in $M_{\pi}$ so that the overall order is $\mathcal{O}\left(M_{\pi}^{2}\right)$ for $\omega=M_{\pi}$, one obtains the original LEG 60]. The mathematical origin of the problem is an illicit interchange of limits : expanding a function $f\left(\omega, M_{\pi}\right)$ in the manner just described and setting $\omega=M_{\pi}$ at the end will in general not lead to the same result as an expansion of $f\left(M_{\pi}, M_{\pi}\right)$ to the same order in $M_{\pi}$. Although it is shown in Ref. [60] that one can recover the correct LET by a resummation of the series to all orders 
in $\omega$, there is in general no guarantee that off-shell manipulations produce the correct result. A simple, but instructive example is to consider the elastic $\pi \pi$ scattering amplitude to lowest order, $\mathcal{O}\left(p^{2}\right)$, both in CHPT and in the linear $\sigma$ model. Although the amplitudes agree on-shell, they disagree in general off-shell. In fact, one can obtain very different forms for the off-shell amplitude by redefining the pion field. While one would normally not employ such redefinitions in the linear model (seemingly destroying renormalizability), any choice of pion field is equally acceptable in CHPT which is based on an intrinsically non-renormalizable quantum field theory. Off-shell manipulations are dangerous and may lead to incorrect results. The literature on applications of current algebra techniques abounds with examples. Concerning the phenomenology of neutral pion photoproduction off nucleons, I refer the reader to the extensive discussion in ref. [44. There, it is shown that the electric dipole amplitude $E_{0+}$ is indeed not a good testing ground of the chiral dynamics but rather there exist LETs in the $P$-waves, which can be used to help analyze the data. An example is given in the next section.

The last example I want to treat is the case of two-pion photoproduction. At threshold, the two-pion photoproduction current matrix element can be decomposed into amplitudes as follows (to first order in $e$ and in the gauge $\epsilon_{0}=0$ ):

$$
T \cdot \epsilon=\chi_{f}^{\dagger}\left\{i \vec{\sigma} \cdot(\vec{\epsilon} \times \vec{k})\left[M_{1} \delta^{a b}+M_{2} \delta^{a b} \tau^{3}+M_{3}\left(\delta^{a 3} \tau^{b}+\delta^{b 3} \tau^{a}\right)\right]\right\}
$$

with $\chi_{i, f}$ two-component Pauli and isospinors. Here, I am only interested in the first non-vanishing contributions to $M_{2,3}$, given by some tree diagrams. So we have $N_{\gamma}=1$, $L=0$ and only lowest order mesonic vertices $(d=2)$, i.e.

$$
D_{L}=\sum_{d}(d-1) N_{d}^{M B}
$$

Tree diagrams with lowest order vertices from $\mathcal{L}_{\pi N}^{(1)}$ all vanish due to the threshold selection rules $\epsilon \cdot v=\epsilon \cdot q_{i}=0, S \cdot q_{i}=0$ and $v \cdot\left(q_{1}-q_{2}\right)=0$. The first non-vanishing contribution comes from tree diagrams with insertions from $\mathcal{L}_{\pi N}^{(2)}$, in particular the expansion of $f_{\mu \nu}^{+}$ from eq.(50) leads to a $\gamma \pi \pi N N$ vertex proportional to $\stackrel{\circ}{\kappa}_{V}$ so that

$$
M_{2}=-2 M_{3}=\frac{e}{4 \stackrel{\circ}{m} F^{2}}\left(2 \stackrel{\circ}{g}_{A}^{2}-1-\stackrel{\circ}{\kappa}_{V}\right)+\mathcal{O}(p)=\frac{e}{4 m F_{\pi}^{2}}\left(2 g_{A}^{2}-1-\kappa_{V}\right)+\mathcal{O}(p)
$$

The full one-loop results can be found in ref. 61]. It is amusing to note that this particular vertex has been overlooked in other derivations of these LETs [62 663 simply because not the most general effective $\pi N$ Lagrangian at order $p^{2}$ was used. 


\section{Confronting the data}

As already stated in the introduction, most of the precision data on the nucleon at low energies come from processes involving real or virtual photons such as pion photo- and electroproduction as well as Compton scattering. This is mostly due to the advent of the $\mathrm{CW}$ accelerators such as MAMI at Mainz and improved detector technology. It is worth to stress that there are on-going activities in these fields also at NIKHEF (Amsterdam), SAL (Saskatoon), BATES (MIT), ELSA (Bonn) and other places. Other precise data come from atomic energy shifts at PSI (Villigen) and there is also a tremendous amount of threshold data for $\pi N \rightarrow \pi \pi N$ from such places like TRIUMF (Vancouver). Just from the beginning I would like to stress that one should not see these different experiments and data in isolation but that they are rather intimately connected. For example, the imaginary parts of the various multipoles in pion photo- and electroproduction are proportional to the respective pion-nucleon scattering phase shifts via the Fermi-Watson final state theorem. As an example, let me give the imaginary part of the electric dipole amplitude in neutral pion photoproduction off protons,

$$
\operatorname{Im} E_{0+}^{\pi^{0} p}=\operatorname{Re} E_{0+}^{(0)} \tan \left(\delta_{1 / 2}\right)+\frac{1}{3} \operatorname{Re} E_{0+}^{(1 / 2)} \tan \left(\delta_{1 / 2}\right)+\frac{2}{3} \operatorname{Re} E_{0+}^{(3 / 2)} \tan \left(\delta_{3 / 2}\right),
$$

with $\delta_{1 / 2,3 / 2}$ the pion-nucleon $\mathrm{S}$-wave phase shifts for total isospin $1 / 2$ and $3 / 2$, respectively. Also, various contact terms show up in different reactions. For example, the LECs $c_{1,2,3}$ which can be determined in $\pi N \rightarrow \pi N$ [37 show up in the order $q^{4}$ calculation of the nucleons' electromagnetic polarizabilities [25] from non-vanishing insertions of $\mathcal{L}_{\pi N}^{(2)}$. This should always be kept in mind. For a nice and instructive flow-chart giving the links between low-energy pion experiments I refer the reader to Fig.7.33-1 in the monograph of de Benedetti [64].

Let us now consider some of the data with electromagnetic and weak probes which have become available over the last few years. In table 2, I summarize these in comparison to the chiral predictions. Clearly, there are more measured quantities, but I have only included the ones which (a) can be predicted with some accuracy and (b) for which some solid data exist. The most notabel absentee from this table is the electric dipole amplitude for $\gamma p \rightarrow \pi^{0} p$. First, as discussed in length in refs. 65] [44, no solid chiral prediction exists at present and second, the empirical determination hinges on a few empirical points and some more or less justified assumptions about the $\mathrm{P}$-waves. This reaction has been remeasured at MAMI and SAL and one should wait for the analysis of these new data. Quite in contrast to common folklore, there exist a set of LETs for the slopes of the $\mathrm{P}$-waves $P_{1,2}=3 E_{1+} \pm M_{1+} \mp M_{1-}$ at threshold, e.g.

$$
\frac{1}{|\vec{q}|} P_{1, \text { thr }}^{\pi^{0} p}=\frac{e g_{\pi N}}{8 \pi m^{2}}\left\{1+\kappa_{p}+\mu\left[-1-\frac{\kappa_{p}}{2}+\frac{g_{\pi N}^{2}}{48 \pi}(10-3 \pi)\right]+\mathcal{O}\left(\mu^{2}\right)\right\} .
$$


Numerically, this translates into

$$
\frac{1}{|\vec{q}|} P_{1, \mathrm{thr}}^{\pi^{0} p}=0.512(1-0.062) \mathrm{GeV}^{-2}=0.480 \mathrm{GeV}^{-2}
$$

which is given in table 2 in units which are more used in the literature. For a discussion of the other quantities, I refer to the quoted references.

\begin{tabular}{|c|c|c|c|c|c|c|}
\hline Observable & Prediction & Order & Ref. & Data & Ref. & Units \\
\hline $\bar{\alpha}_{p}$ & $10.5 \pm 2.0$ & $q^{4}$ & 25 & $10.4 \pm 0.6$ & [6] & $10^{-4} \mathrm{fm}^{3}$ \\
\hline $\bar{\beta}_{p}^{P}$ & $3.5 \pm 3.6$ & $q^{4}$ & 25 & $3.8 \mp 0.6$ & 俩 & $10^{-4} \mathrm{fm}^{3}$ \\
\hline $\bar{\alpha}_{n}$ & $13.4 \pm 1.5$ & $q^{4}$ & 25 & $12.3 \pm 1.3$ & 5 & $10^{-4} \mathrm{fm}^{3}$ \\
\hline $\bar{\beta}_{n}$ & $7.8 \pm 3.6$ & $q^{4}$ & 25 & $3.5 \mp 1.3$ & 5 & $10^{-4} \mathrm{fm}^{3}$ \\
\hline$E_{0+, \text { thr }}^{\pi^{+} n}$ & 28.4 & $q^{3}$ & 50 & $27.9 \pm 0.5$ & 66 & $10^{-3} / M_{\pi^{+}}$ \\
\hline$E_{0+, \text { thr }}^{\pi^{-}}$ & -31.1 & $q^{3}$ & 50 & $-31.4 \pm 1.3$ & 66 & $10^{-3} / M_{\pi^{+}}$ \\
\hline$P_{1, \mathrm{thr}}^{\pi^{0} p}$ & 10.9 & $q^{3}$ & 44 & $8.8 \pm 0.6$ & 67 & $|\vec{q}||\vec{k}| 10^{-3} / M_{\pi^{+}}^{3}$ \\
\hline$\left|L_{0+, \mathrm{thr}}^{\pi^{0} p}\right|^{2}$ & 0.20 & $q^{3}$ & 68 & $0.13 \pm 0.05$ & 3 & $\mu \mathrm{b}$ \\
\hline$g_{P}$ & $8.44 \pm 0.23$ & $q^{3}$ & 69 & $8.7 \pm 1.6$ & 70 & - \\
\hline
\end{tabular}

Table 2: Chiral predictions for electroweak probes compared to experimental data. Given are the electric $(\bar{\alpha})$ and magnetic $(\bar{\beta})$ polarizabilities of the proton and the neutron, the electric dipole amplitudes for charged pion photoproduction, the $\mathrm{P}$-wave $P_{1}$ for neutral pion photoproduction off protons, the longitudinal S-wave multipole for neutral pion electroproduction off protons and the induced pseudoscalar coupling constant, $g_{P}$, measured in muon capture. In some cases, the theoretical uncertainty of the calculation has been estimated and the respective order $\left(q^{3}\right.$ or $\left.q^{4}\right)$ is also given.

There is, of course, ample of data on elastic pion-nucleon scattering and on $\pi N \rightarrow \pi \pi N$ in the threshold region. For a review on the status in $\pi N \rightarrow \pi N$, I refer to Sainio and Höhler [72] [73]. Here, I briefly want to mention some work concerning the chiral corrections to these processes in the framework of HBCHPT. Consider first the on-shell forward $\pi N$ scattering amplitude [37,

$$
T=\delta^{a b} T^{+}(\omega)+i \epsilon^{b a c} \tau^{c} T^{-}(\omega)
$$

with $\omega=v \cdot q$ the pion energy. At threshold, $\omega=M_{\pi}$, we have

$$
T^{-}\left(M_{\pi}\right)=4 \pi(1+\mu) a^{-}=\frac{4}{3} \pi(1+\mu)\left(a_{1 / 2}-a_{3 / 2}\right),
$$


with $\mu=M_{\pi} / m$ and $a_{1 / 2,3 / 2}$ are the $\mathrm{S}$-wave scattering lengths in the channels with total isospin $1 / 2$ and $3 / 2$, respectively. The chiral expansion of $T^{-}\left(M_{\pi}\right)$ reads:

$$
T^{-}\left(M_{\pi}\right)=\frac{M_{\pi}}{2 F_{\pi}^{2}}+\frac{g_{\pi N}^{2} M_{\pi}^{3}}{8 m^{4}}+\frac{M_{\pi}^{3}}{16 \pi^{2} F_{\pi}^{4}}\left(1-2 \ln \frac{M_{\pi}}{\lambda}\right)+\frac{g_{\pi N}^{2} M_{\pi}^{3}}{2 m^{2} m_{\Delta}^{2}}\left(Z-\frac{1}{2}\right)^{2}+\mathcal{O}\left(M_{\pi}^{5}\right) .
$$

It is remarkable that there are no corrections of order $M_{\pi}^{2}$ and $M_{\pi}^{4}$. The various terms in eq. (98) are the current algebra prediction [74, the expansion of the nucleon pole term, the one-loop and the counterterm contribution form $\mathcal{L}_{\pi N}^{(3)}$, respectively. $\lambda$ is the scale introduced in dimensional regularization and the pertinent LEC has been estimated by $\Delta$ exchange (there is also a small contribution from the Roper which I drop). At the resonance scale $\lambda=m_{\Delta}$, we find

$$
T^{-}\left(M_{\pi}\right)=\left(1.59+0.02+0.24+0.04(Z-1 / 2)^{2}\right) \mathrm{fm}=1.87 \mathrm{fm}
$$

for the $\Delta N \pi$ off-shell parameter $Z=-1 / 4$. This agrees nicely with the empirical value of $T^{-}\left(M_{\pi}\right)=(1.86 \pm 0.04) \mathrm{fm}$ [71]. If one chooses $\lambda=m$, the contribution from the loops drops to $0.22 \mathrm{fm}$. The main message here is that only the loop correction at order $M_{\pi}^{3}$ can close the gap between the Weinberg-Tomozawa prediction [74 [75] and the empirical value. Furthermore, the contact term contribution is small so uncertainties in the corresponding LECs get washed out by a small prefactor.

The last issue I want to address is the reaction $\pi N \rightarrow \pi \pi N$. Here, ample new and rather accurate data in the threshold region exist [76] [77] [78] [79] [80] but the one-loop calculation has not yet been performed. However, in ref. [81] the first corrections to the lowest order where considered. At threshold, the T-matrix can be written in terms of two amplitudes $D_{1}$ and $D_{2}$,

$$
T=i \vec{\sigma} \cdot \vec{k}\left[D_{1}\left(\tau^{b} \delta^{a c}+\tau^{c} \delta^{a b}\right)+D_{2} \tau^{a} \delta^{b c}\right]
$$

where $a, b, c$ are isospin indices of the pions and $\vec{k}$ denotes the three momentum of the incoming pion. One can then derive LETs for $D_{1,2}$ 81

$$
\begin{gathered}
D_{1}=\frac{g_{A}}{8 F_{\pi}^{3}}\left(1+\frac{7 M_{\pi}}{2 m}\right)+\mathcal{O}\left(M_{\pi}^{2}\right)=2.4 \mathrm{fm}^{3} \\
D_{2}=-\frac{g_{A}}{8 F_{\pi}^{3}}\left(3+\frac{17 M_{\pi}}{2 m}\right)+\mathcal{O}\left(M_{\pi}^{2}\right)=-6.8 \mathrm{fm}^{3}
\end{gathered}
$$

Now the amplitude $D_{1}$ is exclusively sensitive to the two-pion final state with total isospin $I_{\pi \pi}=2$ whereas $D_{2}$ is dominated by the isospin zero $\mathrm{S}$-wave. Consequently, one expects a small and a sizeable correction at order $M_{\pi}^{2}$ for $D_{1}$ and $D_{2}$, in order. This is corroborated by the calculation of the imaginary part of the one-loop diagrams which give a non-zero imaginary part at that order as detailed in ref. [81]. A best fit to the data from threshold to $20 \mathrm{MeV}$ above (for the pion kinetic energy) gives the empirical values

$$
D_{1}=2.2 \mathrm{fm}^{3}, \quad D_{2}=-8.8 \mathrm{fm}^{3},
$$

which are $9 \%$ and $29 \%$ off the LET predictions, eqs.(101,102). These deviation show exactly the trend discussed before. Clearly, it is mandatory to complete the full order $q^{3}$ calculation before any further conclusions can be drawn. 


\section{Problems and open questions}

Here, I will list a few topics which deserve further study. This list should neither be considered complete nor does the ordering imply any priority.

(i) In the two-flavor sector, we have to perform the one-loop calculation for $\pi N \rightarrow \pi \pi N$ (to order $q^{3}$ or even better, $q^{4}$ ). At present, only tree level calculations and the first corrections from $\mathcal{L}_{\pi N}^{(2)}$ are available. This is in marked contrast to the many existing rather precise data. What one finally wants to learn is to what precision these threshold pion production data encode information about the low energy elastic $\pi \pi$ scattering amplitude. Presently available determimations of the S-wave $\pi \pi$ scattering lenghts from these data should only be considered as estimates.

(ii) More precise data to which HBCHPT can be applied are needed. This would then allow for a systematic study of the LECs appearing in $\mathcal{L}_{\pi N}^{(3,4)}$ and to judge the valitidy (quality) of the resonance saturation principle which is often used to get a handle on the LECs. This calls for a joint effort of the experimenters and the theoreticians.

(iii) In the three flavor sector, despite lots of mumbling and talking, we do not yet have a consistent picture or a set of complete (and hopefully accurate) calculations to draw decisive conclusions. There exist many data and more are coming, as an example let me just mention the accurate threshold kaon photoproduction ones from ELSA or the proposals to measure the hyperon polarizabilities at Fermilab and CERN.

(iv) Jenkins and Manohar first advocated to supplement the EFT of the ground state baryons and Goldstone bosons by the spin-3/2 decuplet [82] [26]. This approach has been taken up in quite a few papers thereafter. If one thinks of extending calculations like $\pi N \rightarrow \pi N$ through the $\Delta$ region, this is certainly unavoidable. However, what is missing is a set of complete calculations for threshold observables from which one could (a) assess the accuracy of the approach and (b) the residual octet-decuplet mass difference has to be treated in a more systematic fashion.

(v) The extension of the CHPT approach to systems of two or more nucleons has only begun. Despite some theoretical problems (the power counting only applies to the subset of irreducible diagrams), it seems to shed some light on the phenomenology of nuclear forces like e.g. the smallness of three-, four--, .. body forces and the masking of isospin violation in these systems. However, it is mandatory to perform these calculations with all the input which is available from the single baryon sector. This has not yet been done. 


\section{Acknowledgements}

First, I would like to thank the organizers for their kind invitation and hospitality. I am also grateful to Véronique Bernard and Norbert Kaiser for fruitful collaborations and allowing me to present some material before publication. I would like to thank Gerhard Ecker and Jürg Gasser for sharing with me their insight into the chiral dynamics.

\section{References}

[1] E. Mazzucato et al., Phys. Rev. Lett. 57 (1986) 3144

[2] R. Beck et al., Phys. Rev. Lett. 65 (1990) 1841

[3] T.P. Welch et al., Phys. Rev. Lett. 69 (1992) 2761

[4] F. Federspiel et al., Phys. Rev. Lett. 67 (1991) 1511

[5] J. Schmiedmayer et al., Phys. Rev. Lett. 66 (1991) 1015

[6] E.L. Hallin et al., Phys. Rev. C48 (1993) 1497

[7] A. Zieger et al., Phys. Lett. B278 (1992) 34

[8] A. Buchmann, these proceedings

[9] J. Gasser and H. Leutwyler, Ann. Phys. 158 (1984) 142

[10] J. Gasser and H. Leutwyler, Nucl. Phys. B250 (1985) 465

[11] Ulf-G. Meißner, Rep. Prog. Phys. 56 (1993) 903

[12] G. Ecker, Czech. J. Phys. 44 (1994) 405

[13] S. Coleman, "Aspects of Symmetry", Cambridge University Press, Cambridge, 1985

[14] S. Weinberg, Physica 96A (1979) 327

[15] J. Gasser, M.E. Sainio and A. Švarc, Nucl. Phys. B307 (1988) 779

[16] E. Jenkins and A.V. Manohar, Phys. Lett. B255 (1991) 558

[17] H. Leutwyler, Ann. Phys. 235 (1994) 165

[18] S. Coleman, J. Wess and B. Zumino, Phys. Rev. 177 (1969) 2239; C.G. Callan, S. Coleman, J. Wess and B. Zumino, ibid 2247

[19] S. Weinberg, Phys. Rev. 177 (1968) 1568 
[20] V. Bernard, N. Kaiser, J. Kambor and Ulf-G. Meißner, Nucl. Phys. B388 (1992) 315

[21] M. Goldberger and S.B. Treiman, Phys. Rev. 110 (1958) 1478

[22] M. Rho, Phys. Rev. Lett. 66 (1991) 1275

[23] S. Weinberg, Phys. Lett. B251 (1990) 288

[24] S. Weinberg, Nucl. Phys. B363 (1991) 3

[25] V. Bernard, N. Kaiser, Ulf-G. Meißner and A. Schmidt, Phys. Lett. B319 (1993) 269; Z. Phys. A348 (1994) 317

[26] E. Jenkins and A.V. Manohar, in "Effective Field Theories of the Standard Model", ed. Ulf-G. Meißner, World Scientific, Singapore, 1992

[27] J.C. Collins, "Renormalization", Cambridge University Press, Cambridge, 1984

[28] P. Langacker and H. Pagels, Phys. Rev. D8 (1975) 4595

[29] N.H. Fuchs, H. Sazdjian and J. Stern, Phys. Lett. B269 (1991) 183;

J. Stern, H. Sazdjian and N.H. Fuchs, Phys. Rev. 47 (1993) 3814

[30] J. Gasser, Ann. Phys. 136 (1981) 62

[31] E. Jenkins, Nucl. Phys. B368 (1992) 190

[32] E. Jenkins and A.V. Manohar, Phys. Lett. B281 (1992) 336

[33] V. Bernard, N. Kaiser and Ulf-G. Meißner, Z. Phys. C60 (1993) 111

[34] R.F. Lebed and M.A. Luty, Phys. Lett. B29 (1994) 479

[35] S. Mallik, "Massive States in Chiral Perturbation Theory", Saha Institute preprint, 1994

[36] M.K. Banerjee and J. Milana, "Baryon Mass Splittings in Chiral Perturbation Theory", preprint UMPP 95-058, 1994

[37] V. Bernard, N. Kaiser and Ulf-G. Meißner, Phys. Lett. B309 (1993) 421

[38] V. Bernard, N. Kaiser and Ulf-G. Meißner, "Chiral Symmetry in Nuclear Physics", in preparation

[39] C. Ordonez and U. van Kolck, Phys. Lett. B291 (1992) 459; C. Ordonez, L. Ray and U. van Kolck, Phys. Rev. Lett. 72 (1994) 1982; U. van Kolck, Phys. Rev. C49 (1994) 2932

[40] U. van Kolck, thesis, Univ. of Texas at Austin, 1993 
[41] G. Ecker, J. Gasser, A. Pich and E. de Rafael, Nucl. Phys. 321 (1989) 311; J.F. Donoghue, C. Ramirez and G. Valencia, Phys. Rev. D39 (1989) 1947

[42] Ulf-G. Meißner, "Aspects of Nucleon Chiral Perturbation Theory", talk given at the Workshop on Chiral Dynamics : Theory and Experiment, MIT, Cambridge, July 1994, preprint CRN 94/44

[43] G. Ecker. Phys. Lett. B336 (1994) 508

[44] V. Bernard, N. Kaiser and Ulf-G. Meißner, "Neutral Pion Photoproduction off Nucleons Revisited", preprint CRN 94-62 and TK 94 18, 1994

[45] G. Ecker and Ulf-G. Meißner, "What is a Low-Energy Theorem?", prperint CRN 94-52 and UWThPh-1994-33, 1994

[46] F. Low, Phys. Rev. 96 (1954) 1428;

M. Gell-Mann and M.L. Goldberger, ibid. 1433

[47] S. Weinberg, Phys. Rev. Lett. 18 (1967) 188

[48] V. Bernard, N. Kaiser and Ulf-G. Meißner, Nucl. Phys. B373 (1992) 346

[49] V. Bernard, J. Gasser, N. Kaiser and Ulf-G. Meißner, Phys. Lett. B268 (1991) 291

[50] V. Bernard, N. Kaiser and Ulf-G. Meißner, Nucl. Phys. B383 (1992) 442

[51] N.M. Kroll and M.A. Ruderman, Phys. Rev. 93 (1954) 233

[52] A.I. Vainsthein and V.I. Zakharov, Yad. Fiz. 12 (1970) 610 (Sov. J. Nucl. Phys. 12 (1971) 333); Uspekhi Fiz. Nauk 100 (1970) 225 (Sov. Phys. Uspekhi 13 (1970) 73); Nucl. Phys. B36 (1972) 589

[53] P. de Baenst, Nucl. Phys. B24 (1970) 633

[54] A.M. Bernstein and B.R. Holstein, Comments Nucl. Part. Phys. 20 (1991) 197

[55] H.W.L. Naus, J.H. Koch and J.L. Friar, Phys. Rev. C41 (1990) 2852

[56] H.W.L. Naus, Phys. Rev. C43 (1991) R365

[57] A.N. Kamal, Int. J. Mod. Phys. A6 (1991) 263

[58] H.W.L. Naus, Phys. Rev. C44 (1991) 531

[59] S. Scherer, J.H. Koch and J.L. Friar, Nucl. Phys. A552 (1993) 515

[60] R.M. Davidson, Phys. Rev. C47 (1993) 2492

[61] V. Bernard, N. Kaiser and Ulf-G. Meißner, Nucl. Phys. A580 (1994) 475 
[62] R. Dahm and D. Drechsel, in Proc. Seventh Amsterdam Mini-Conference, eds. H.P. Blok, J.H. Koch and H. De Vries, Amsterdam, 1991

[63] M. Benmerrouche and R. Tomusiak, Phys. Rev. Lett. 73 (1994) 400

[64] S. DeBenedetti, "Nuclear Interactions", John Wiley and Sons, New York, 1964

[65] V. Bernard, "Threshold Pion Photo- and Electroproduction in Chiral Perturbation Theory", talk given at the Workshop on Chiral Dynamics : Theory and Experiment, MIT, Cambridge, July 1994, preprint CRN 94/45, hep-ph/9408323

[66] J. P. Burg, Ann. Phys. (Paris) 10 (1965) 363

[67] D. Drechsel and L. Tiator, J. Phys. G: Nucl. Part. Phys. 18 (1992) 449

[68] V. Bernard, T.-S. H. Lee, N. Kaiser and Ulf-G. Meißner, Phys. Rep. 246 (1994) 315

[69] V. Bernard, N. Kaiser and Ulf-G. Meißner, Phys. Rev. D50 (1994) in print

[70] G. Bardin et al., Phys. Lett. B104 (1981) 320

[71] G. Höhler, Landolt-Börnstein vol. 9b2, H. Schopper (ed.), Springer, Berlin, 1983

[72] M. E. Sainio, "Pion-Nucleon Sigma Term", talk given at the Workshop on Chiral Dynamics : Theory and Experiment, MIT, Cambridge, July 1994, preprint HU-TFT94-40

[73] G. Höhler, "Tests of Predictions from Chiral Perturbation Theory for $\pi N$ Scattering", talk given at the Workshop on Chiral Dynamics : Theory and Experiment, MIT, Cambridge, July 1994, Karlsruhe preprint

[74] S. Weinberg, Phys. Rev. Lett. 17 (1966) 616

[75] Y. Tomozawa, Nuovo Cim. 46A (1966) 707

[76] D. Pocancic et al., Phys. Rev. Lett. 72 (1994) 1156

[77] H. Burkhardt and J. Lowe, Phys. Rev. Lett. 67 (1991) 2622

[78] M.E. Sevior et al., Phys. Rev. Lett. 66 (1991) 2569

[79] G. Kernel et al., Z. Phys. C48 (1990) 201

[80] J. Lowe et al., Phys. Rev. C44 (1991) 956

[81] V. Bernard, N. Kaiser and Ulf-G. Meißner, Phys. Lett. B332 (1994) 415

[82] E. Jenkins and A.V. Manohar, Phys. Lett. B259 (1991) 353 